\title{
Mathematical Formulation of the No-Go Theorem in Horndeski Theory ${ }^{+}$
}

\author{
Sergey Mironov ${ }^{1,2}$ \\ 1 Institute for Nuclear Research of the Russian Academy of Sciences, 60th October Anniversary Prospect, 7a, \\ 117312 Moscow, Russia; sa.mironov_1@physics.msu.ru \\ 2 Institute for Theoretical and Experimental Physics, Bolshaya Cheriomyshkinskaya, 25, \\ 117218 Moscow, Russia \\ + This paper is based on the talk at the 7th International Conference on New Frontiers in Physics (ICNFP 2018), \\ Crete, Greece, 4-12 July 2018.
}

Received: 31 December 2018; Accepted: 29 January 2019; Published: 2 February 2019

\begin{abstract}
We present a brief mathematical-like formulation of the no-go theorem, useful for bouncing and wormhole solutions in Horndeski theory. The no-go theorem is almost identical in the cases of flat FLRW geometry and static, spherically symmetric setting, hence, we generalize the argument of the theorem so that it has consise and universal form. We also give a strict mathematical proof of the no-go argument.
\end{abstract}

Keywords: stability conditions; strong coupling; ghosts and gradient instabilities; bouncing cosmologies and wormhole solutions

\section{Introduction and Summary}

Let us study the most general scalar-tensor theory with second-order equations of motion, namely, the Horndeski theory [1]. Despite the fact that the Null Energy condition (NEC) can be violated in a healthy way within this theory (see e.g., Ref. [2]), it turns out that non-trivial solutions without singularities, both cosmological and gravitational, still involve instabilities or strong coupling, provided that one considers the entire space-time manifold. The inevitable presence of pathologies was shown for both static, spherically symmetric, and time-dependent homogeneous solutions. The corresponding statements were formulated as no-go theorems [3-6]. Generally, one finds that the quadratic action for linearized perturbations in Horndeski theory necessarily acquire wrong signs or zeroes in coefficients (in some cases, the latter fact signifies a strong coupling regime). Let us illustrate the issue of stability within a cosmological setup. For a spatially flat metric of the FLRW type, the quadratic action in unitary gauge (i.e., with vanishing scalar field perturbations) is as follows $[7,8]$ :

$$
S=\int \mathrm{d} t \mathrm{~d}^{3} x a^{3}\left[\frac{\mathcal{G}_{\mathcal{T}}}{8}\left(\dot{h}_{i k}^{T}\right)^{2}-\frac{\mathcal{F}_{\mathcal{T}}}{8 a^{2}}\left(\partial_{l} h_{i k}^{T}\right)^{2}+\mathcal{G}_{\mathcal{S}} \dot{\zeta}^{2}-\mathcal{F}_{\mathcal{S}} \frac{(\nabla \zeta)^{2}}{a^{2}}\right]
$$

where $a$ is a scale factor, $h_{i k}^{T}$ stands for transverse, traceless tensor perturbations, while $\zeta$ is the only dynamical degree of freedom in the scalar sector. Let us note that the quadratic action above is of the most general form in the FLRW background of the Horndeski theory, modulo the specified gauge choice. The coefficients $\mathcal{G}_{\mathcal{T}}, \mathcal{F}_{\mathcal{T}}, \mathcal{G}_{\mathcal{S}}$, and $\mathcal{F}_{\mathcal{S}}$ are some expressions in terms of Lagrangian functions, which are constrained by the stability requirement of the linearized theory. Positive $\mathcal{G}_{\mathcal{T}}$ and $\mathcal{G}_{\mathcal{S}}$ guarantee the absence of ghosts in tensor and scalar sectors, while positive $\mathcal{F}_{\mathcal{T}}$ and $\mathcal{F}_{\mathcal{S}}$ are required for the absence of gradient instabilities. Now, the Infinum of these coefficients naively corresponds to a strong coupling scale, which is why we require $\mathcal{G}_{\mathcal{T}}, \mathcal{F}_{\mathcal{T}}, \mathcal{G}_{\mathcal{S}}$, and $\mathcal{F}_{\mathcal{S}}$ in the following to be greater than some positive constant value $\epsilon$. 
The behaviour of perturbations in a static, spherically symmetric setup is in full analogy to the cosmological one and the corresponding stability analysis gives similar expressions for the constraints. Let us note that we do not give a specific form of any coefficients involved in the quadratic action, since the no-go theorem relies on the general behaviour of the structures involved only. The only important aspect about the coefficients $\mathcal{G}_{\mathcal{T}}, \mathcal{F}_{\mathcal{T}}, \mathcal{G}_{\mathcal{S}}$, and $\mathcal{F}_{\mathcal{S}}$ is that both $\mathcal{G}_{\mathcal{T}}$ and $\mathcal{F}_{\mathcal{T}}$ are linear combinations of Lagrangian functions and, hence, must be regular. We also use the non-trivial relations between the $\mathcal{G}_{\mathcal{S}}, \mathcal{F}_{\mathcal{S}}$ and $\mathcal{G}_{\mathcal{T}}, \mathcal{F}_{\mathcal{T}}$ in a setup of the theorem.

In the following section, we formulate the no-go theorem using the notations of the cosmological setup, but as it was explicitly shown in Ref. [9], the structure of stability conditions for the spherically symmetric case is the same; hence, the argument below applies to both homogeneous and inhomogeneous settings. The motivation for restating the theorem in a formal way was to have it in a clear and concise form in order to avoid any future misunderstanding of the concept behind it. We emphasise that we consider strong coupling, i.e., $\mathcal{G}_{\mathcal{T}} \rightarrow 0$, to be a dissatisfying feature. In the next section, we show that due to the particular constraints imposed on the coefficients of the quadratic action in the Horndeski theory, any complete, healthy regular solution has $\mathcal{G}_{\mathcal{S}}$ and $\mathcal{F}_{\mathcal{S}}$, which are singular everywhere.

\section{No-Go Theorem}

Theorem 1. Set up: We consider functions on $\mathbb{R}^{1}$ with the following relations (it is exactly the case of the general Horndeski theory):

$$
\begin{aligned}
& \mathcal{G}_{\mathcal{S}}=\frac{\Sigma \mathcal{G}_{\mathcal{T}^{2}}}{\Theta^{2}}+3 \mathcal{G}_{\mathcal{T}}, \\
& \mathcal{F}_{\mathcal{S}}=\frac{1}{a} \frac{\mathrm{d} \xi}{\mathrm{d} t}-\mathcal{F}_{\mathcal{T}}, \\
& \xi=\frac{a \mathcal{G}_{\mathcal{T}}{ }^{2}}{\Theta} .
\end{aligned}
$$
Assumptions:
(1) $\mathcal{G}_{\mathcal{T}}, \mathcal{F}_{\mathcal{T}}, \Sigma, \Theta$, and a are smooth functions of coordinate $q^{1}$.
(2) $\exists \epsilon>0: \mathcal{G}_{\mathcal{T}}>\mathcal{F}_{\mathcal{T}}>\epsilon, \quad \mathcal{G}_{\mathcal{S}}>\mathcal{F}_{\mathcal{S}}>\epsilon, \quad a>\epsilon$.

Statement:

The only relevant function choice to satisfy the assumptions is $\Theta=0$ everywhere.

Proof. Suppose $\Theta \neq 0$ at some point $P$, due to the continuity $\Theta \neq 0$ in the vicinity of $P$. Let us consider the whole interval where $\Theta \neq 0$. It is limited either by points $q_{ \pm}$where $\Theta=0$ or $q_{ \pm}=\infty$. In this interval, functions $\mathcal{G}_{\mathcal{S}}, \mathcal{F}_{\mathcal{S}}$, and $\xi$ are continuous as well, since:

$$
\frac{\mathrm{d} \xi}{\mathrm{d} t}>a\left(\mathcal{F}_{\mathcal{S}}+\mathcal{F}_{\mathcal{T}}\right)>2 \epsilon
$$

therefore, $\xi \rightarrow \infty$ on the right boundary $\left(q \rightarrow q_{+}\right)$and $\xi \rightarrow-\infty$ on the left boundary $\left(q \rightarrow q_{-}\right)$. Finally, since $\xi$ is continuous, $\exists$ point $P$ where $\xi=0$. This contradicts with positive $\mathcal{G}_{\mathcal{T}}$ and $a$.

Therefore, $\Theta=0$ everywhere.

\section{Discussion}

It should be noted that we did not restrict ourselves to the finite $\mathcal{G}_{\mathcal{S}}$ and $\mathcal{F}_{\mathcal{S}}$. Indeed, taking $\Theta=0$ at a glance results in singular behaviour of both $\mathcal{G}_{\mathcal{S}}$ and $\mathcal{F}_{\mathcal{S}}$ [10-12]. However, it was checked explicitly

1 Coordinate $q$ stands for time coordinate in a cosmological setup and radial coordinate in a static, spherically symmetric case, for instance, in a wormhole setup. 
in Ref. [13] that in the flat FLRW case, the relation $\mathcal{F}_{\mathcal{S}} / \mathcal{G}_{\mathcal{S}}$, which corresponds to the sound speed squared in the scalar sector, remains perfectly finite. The latter fact signifies that the singularities, which arise when $\Theta$ hits zero value, are present only on the level of the linearized equations, but not the solutions. Hence, there is nothing wrong with $\Theta=0$. The statement has its direct analogue in a static, spherically symmetric case-see Ref. [9].

As it was specified in the introduction, the no-go theorem above applies to the Horndeski theory in the homogeneous case with a generally flat FLRW form of metric. In the homogeneous cosmological case, $\Theta \propto H$, where $H$ is the Hubble parameter (see Refs. $[8,10,13]$ for details). It is worth noting that the empty Minkowski space is a special case of the Horndeski theory in a homogeneous setting, and gives the example of a solution where $\Theta=0$ always , since $a(t)=$ const and, hence, $H=0$, while the rest of the terms involved in $\Theta$ vanish. Thus, Minkowski space is an example of a solution which satisfies the no-go theorem.

Generally, the theorem is quite universal when it comes to cosmological applications, since Horndeski theories are the generalization of the numerous scalar-tensor theories with second-order equations of motion. Another direct application of the formulated no-go theorem, as we mentioned earlier, is available in the static, spherically symmetric setting, such as a wormhole setup $[6,14,15]$.

We would also like to briefly comment on possible loopholes here. As $\epsilon$, which is involved in the no-go assumptions, is a strong coupling scale, we require it to be strongly positive-yet one can consider asymptotically strong coupling but make sure that scale of the classical evolution stays beyond the strong coupling scale. Another possibility is to consider geodesically incomplete solutions, i.e., asymptotically singular scale factor $a$. Coordinate $q$ might correspond to time (cosmological scenario, e.g., bounce) or a radial coordinate (spherically symmetric solution, e.g., wormhole). Hence, the no-go theorem indeed summarizes both static and homogeneous cases.

Funding: This research was funded by the Foundation for the Advancement of Theoretical Physics and Mathematics (BASIS) grant and RFBR grants 18-32-00812_a, 18-51-05015_Arm and 17-51-50051_Yaf.

Acknowledgments: The author is indebted to V. Volkova and V. Rubakov for fruitful discussions.

Conflicts of Interest: The author declares no conflict of interest.

\section{References}

1. Horndeski, G.W. Second-order scalar-tensor field equations in a four-dimensional space. Int. J. Theor. Phys. 1974, 10, 363-384. [CrossRef]

2. Rubakov, V.A. The Null Energy Condition and its violation. Phys. Usp. 2014, 57, 128. [CrossRef]

3. Libanov, M.; Mironov, S.; Rubakov, V. Generalized Galileons: Instabilities of bouncing and Genesis cosmologies and modified Genesis. JCAP 2016, 2016, 037. [CrossRef]

4. Kobayashi, T. Generic instabilities of nonsingular cosmologies in Horndeski theory: A no-go theorem. Phys. Rev. D 2016, 94, 043511. [CrossRef]

5. Kolevatov, R.; Mironov, S. Cosmological bounces and Lorentzian wormholes in Galileon theories with an extra scalar field. Phys. Rev. D 2016, 94, 123516. [CrossRef]

6. Evseev, O.A.; Melichev, O.I. No static spherically symmetric wormholes in Horndeski theory. Phys. Rev. D 2018, 97, 124040. [CrossRef]

7. Kobayashi, T.; Yamaguchi, M.; Yokoyama, J. Generalized G-inflation: Inflation with the most general second-order field equations. Prog. Theor. Phys. 2011, 126, 511-529. [CrossRef]

8. Kolevatov, R.; Mironov, S.; Sukhov, N.; Volkova, V. Cosmological bounce and Genesis beyond Horndeski. JCAP 2017, 2017, 038. [CrossRef]

9. Mironov, S.; Rubakov, V.; Volkova, V. More about stable wormholes in beyond Horndeski theory. arXiv 2018, arXiv:1812.07022.

10. Kolevatov, R.; Mironov, S.; Rubakov, V.; Sukhov, N.; Volkova, V. Perturbations in generalized Galileon theories. Phys. Rev. D 2017, 96, 125012. [CrossRef]

11. Mironov, S.; Volkova, V. Properties of perturbations in beyond Horndeski theories. Int. J. Mod. Phys. A 2018, 33, 1850155. [CrossRef] 
12. Ijjas, A. Space-time slicing in Horndeski theories and its implications for non-singular bouncing solutions. JCAP 2018, 2018, 007. [CrossRef]

13. Mironov, S.; Rubakov, V.; Volkova, V. Bounce beyond Horndeski with GR asymptotics and $\gamma$-crossing. JCAP 2018, 2018, 050. [CrossRef]

14. Kobayashi, T.; Motohashi, H.; Suyama, T. Black hole perturbation in the most general scalar-tensor theory with second-order field equations I: The odd-parity sector. Phys. Rev. D 2012, 85, 084025. Erratum: [Phys. Rev. D 2017, 96, 109903]. [CrossRef]

15. Kobayashi, T.; Motohashi, H.; Suyama, T. Black hole perturbation in the most general scalar-tensor theory with second-order field equations II: The even-parity sector. Phys. Rev. D 2014, 89, 084042. [CrossRef]

(C) 2019 by the author. Licensee MDPI, Basel, Switzerland. This article is an open access article distributed under the terms and conditions of the Creative Commons Attribution (CC BY) license (http://creativecommons.org/licenses/by/4.0/). 